\title{
Clinical value of lymph node dissection for stage T1b mid-thoracic esophageal squamous cell carcinoma
}

\author{
Xiaofeng Chen", Yujie Chen", Feng Wang, Shuoyan Liu, Peng Chen, Hao He \\ Fujian Medical University Cancer Hospital, Fujian Cancer Hospital, Fujian Provincial Key Laboratory of Tumor Biotherapy, Department of \\ Thoracic Tumor Surgery, Fujian Cancer Hospital, Fuzhou, China \\ Contributions: (I) Conception and design: F Wang, X Chen; (II) Administrative support: F Wang, S Liu; (III) Provision of study materials or patients: \\ X Chen, Y Chen; (IV) Collection and assembly of data: Y Chen; (V) Data analysis and interpretation: X Chen, Y Chen; (VI) Manuscript writing: All \\ authors; (VII) Final approval of manuscript: All authors. \\ \#These authors contributed equally to this work. \\ Correspondence to: Feng Wang. Fujian Provincial Key Laboratory of Tumor Biotherapy, Department of Thoracic Tumor Surgery, Fujian Medical \\ University Cancer Hospital \& Fujian Cancer Hospital, 7F, Surgery Building, Fujian Cancer Hospital, No.420, Fuma Road, Fuzhou 350014, China. \\ Email: wfmd120@163.com.
}

\begin{abstract}
Background: Surgery remains the mainstay of treatment for esophageal squamous cell carcinoma (ESCC), during which lymph node (LN) dissection, especially recurrent laryngeal nerve (RLN) LN dissection, is particularly important and challenging. This study aimed to investigate the LN metastasis of stage T1b midthoracic ESCC and explore the clinical value of RLN LN dissection.

Methods: The clinicopathological data of 254 patients with stage T1b mid-thoracic ESCC who underwent the McKeown procedure ("tri-incisional esophagectomy") and three-field LN dissection (3FD) at Fujian Cancer Hospital from January 2010 to December 2015 were retrospectively analyzed. The value of LN dissection (especially RLN LNs) was evaluated by calculating the metastasis rate of each LN station. The efficacy index (EI) of the dissection was calculated by multiplying the frequency (\%) of metastases to a station and the 5 -year survival rate (\%) of patients with metastases to that station, and then dividing by 100 .

Results: The stage T1b mid-thoracic ESCC had the highest rate of metastasis in the paracardiac LNs (4.3\%), followed by RLN LNs (2.8\%) and the left gastric artery LNs (2.8\%). The 5-year survival rate was highest in patients who received lesser gastric curvature LN dissection (100\%), followed by patients who underwent right RLN LN dissection (80\%), and was 50\% in patients who had undergone dissection of the left RLN LNs, upper paraesophageal LNs, subcarinal LNs, and left gastric artery LNs, respectively. In addition, dissection of the right RLN LNs had the highest EI value (2.2), followed by the dissection of LNs along the lesser curvature of the stomach (1.6) and left gastric artery LNs (1.4).

Conclusions: Right RLN LNs have a metastasis rate only lower than that of the paracardiac LNs, but could be the most valuable location for performing dissection.
\end{abstract}

Keywords: Esophageal cancer; lymph node dissection (LN dissection); recurrent laryngeal nerve lymph nodes (RLN LNs); T1b stage

Submitted May 18, 2021. Accepted for publication Aug 18, 2021.

doi: 10.21037/jgo-21-339

View this article at: https://dx.doi.org/10.21037/jgo-21-339

\section{Introduction}

Esophageal cancer is the sixth leading cause of cancerrelated deaths worldwide due to its high malignant potential and poor prognosis (1). Biologically, the malignancy has evolved from predominantly squamous cell carcinoma (SCC) to adenocarcinoma. Surgery remains the mainstay of treatment for esophageal cancer, and its efficacy has dramatically improved in recent years with advances in 
Table 1 General data of the study subjects

\begin{tabular}{lc}
\hline Basic features & No. (interquartile range or \%) \\
\hline Sample size & 254 \\
Age (years) & $58.0(51.0-63.0)$ \\
Gender & \\
Male & $192(73.2)$ \\
Female & $62(26.8)$ \\
Tumor embolism & $29(11.4)$ \\
\hline
\end{tabular}

surgical procedures and perioperative management. Based on the frequency and extent of lymph node (LN) metastasis and its significance to survival, controlling $\mathrm{LN}$ metastasis is a reasonable treatment strategy. Therefore, an extended $\mathrm{LN}$ dissection such as three-field $\mathrm{LN}$ dissection (3FD) is often mandatory. Numerous reports have demonstrated the superiority of 3FD over two-field dissection. However, the need for 3FD remains controversial due to its large surgical extent, challenging maneuvers, and frequent postoperative complications (especially hoarseness and aspiration pneumonia following dissection of left and right laryngeal recurrent nerve (LRN) LNs (2).

Stage T1a esophageal cancer is confined to the mucosal layer and stage T1b lesions are confined to the submucosal layer. Currently, most guidelines recommend endoscopic treatment for stage Ia esophageal cancer, whereas surgery is the main treatment for stage Ib lesions. Since stage T1b esophageal cancer is an early localized lesion, whether patients should undergo 3FD or which LN groups should be removed have become popular research topics. In this study, we retrospectively analyzed the clinicopathological data of 254 patients with stage T1b mid-thoracic esophageal squamous cell carcinoma (ESCC) who underwent the McKeown procedure ("tri-incisional esophagectomy") and 3FD at Fujian Cancer Hospital from January 2010 to December 2015, with the aim of identifying the LN metastasis of stage T1b ESCC and the clinical value of LN dissection. We present the following article in accordance with the STROBE reporting checklist (available at https:// dx.doi.org/10.21037/jgo-21-339).

\section{Methods}

\section{Subjects}

The study was conducted in accordance with the
Declaration of Helsinki (as revised in 2013). The present study was approved by the Fujian Cancer Hospital Ethics Committee (YKT2021-011-01), and the informed consent was signed by all patients before undergoing surgery. The clinicopathological data of 254 patients with stage T1b mid-thoracic ESCC who underwent the McKeown procedure and 3FD at Fujian Cancer Hospital from January 2010 to December 2015 were retrospectively collected. Of these, 192 were men and 62 were women, aged 51.0 63.0 years (median: 58.0 years) (Table 1). Preoperative barium esophagogram, chest CT, abdominal ultrasound, and gastroscopic pathological biopsy were routinely performed to confirm the diagnosis. All patients were naive to neoadjuvant radiochemotherapy.

\section{Surgical procedures}

All patients underwent the McKeown procedure (subtotal transthoracic esophagectomy, laparotomy, and cervical esophagogastrostomy) and cervico-thoraco-abdominal (three-field) LN dissection (3FD). The extent of LN dissection included: mediastinal LNs [including the right recurrent laryngeal nerve (RLN), left RLN, subcarinal, right and left hilar, and upper/middle/lower esophageal segments]; abdominal LNs (mainly LNs near the gastric cardia, lesser curvature of stomach, and left gastric artery); cervical LNs (mainly the right and left supraclavicular LNs and cervical paratracheoesophageal LNs). Since the cervical paratracheoesophageal LNs were distributed along the left and right RLN and most of them could be removed during chest surgery, the right and left cervical paratracheoesophageal LNs were classified as left and right RLN LNs, respectively. In addition, no metastasis to the right or left hilar LNs was noted in our current study. R0 resection was achieved in all patients, and all cases were followed up for more than 5 years.

\section{Statistical analysis}

Statistical analyses were performed using the SPSS 25.0 software package. The clinical value of LN dissection was evaluated using the efficacy index (EI), which was proposed by Sasako et al. (3). The EI was calculated by multiplying the frequency (\%) of metastases to a station and the 5-year survival rate (\%) of patients with metastases to that station, and then dividing by 100 . A $\mathrm{P}$ value of $<0.05$ was considered statistically significant. 
Table 2 Lymph node metastasis of stage T1 mid-thoracic esophageal squamous carcinoma and value of dissection

\begin{tabular}{|c|c|c|c|}
\hline Site of lymph nodes & Rate of lymph node metastasis & 5 -year survival rate $(\%)$ & El value \\
\hline Left laryngeal recurrent nerve lymph nodes & $5(2.0)$ & 50.0 & 1.0 \\
\hline Right supraclavicular lymph nodes & $0(0.0)$ & - & 0.0 \\
\hline Left supraclavicular lymph nodes & $0(0.0)$ & - & 0.0 \\
\hline Middle para-esophageal lymph nodes & $1(0.4)$ & 0.0 & 0.0 \\
\hline Lower para-esophageal lymph nodes & $0(0.0)$ & - & 0.0 \\
\hline Subcarinal lymph nodes & $2(0.8)$ & 50.0 & 0.4 \\
\hline Paracardiac lymph nodes & $11(4.3)$ & 28.6 & 1.2 \\
\hline
\end{tabular}

The metastasis rate is expressed as [n (\%)]; the 5-year survival rate is the 5-year survival rate of patients with a positive finding in a specific lymph node station (presented as \%); El is the efficacy index of lymph node dissection in a specific station. El, efficacy index.

\section{Results}

The stage T1b mid-thoracic ESCC had the highest rate of metastasis in the paracardiac LNs (4.3\%), followed by the right RLN LNs (2.8\%) and the left gastric artery LNs $(2.8 \%)$. The 5 -year survival rate was highest in patients who received lesser gastric curvature $\mathrm{LN}$ dissection (100\%), followed by patients who underwent right RLN LN dissection ( $80 \%$ ), and was $50 \%$ in patients who had undergone dissection of the left RLN LNs, upper paraesophageal LNs, subcarinal LNs, and left gastric artery LNs, respectively. In addition, dissection of the RLN LNs had the highest EI value (2.0), followed by dissection of the LNs along the lesser curvature of the stomach (1.4) and the LNs near the left gastric artery (1.3) (Table 2).

\section{Discussion}

Despite improved treatment outcomes owing to advances in multidisciplinary therapies, the overall prognosis for esophageal cancer remains poor. Given that the esophagus has abundant lymphatic channels in the submucosa, cancer cells can spread rapidly, and therefore, esophageal cancer can spread easily in early stages (4). 3FD was developed by Japanese surgeons in the 1980s, and is now accepted worldwide as one of the standard surgical approaches for expanded LN dissection (5). However, 3FD may increase postoperative complications. In particular, dissection of the bilateral LRNs and supraclavicular LNs may impair the swallowing and cough reflexes. Also, Yasuda et al. found that laryngeal elevation was significantly impaired after 3FD (6). Nakamura et al. retrospectively compared postoperative dysfunction after two-field versus three-field LN dissection in patients undergoing esophageal cancer surgery, and found that $3 \mathrm{FD}$ was associated with greater postoperative gastrointestinal dysfunction (e.g., decreased physical activity, symptoms of reflux, and passage dysfunction) compared to two-field LN dissection (7). Thus, evidence of the superiority of $3 \mathrm{FD}$ remains unconvincing.

Meanwhile, the McKeown procedure is becoming the dominant procedure in today's esophageal cancer surgery with the adoption of minimally invasive esophagectomy (MIE) over the past two decades, demonstrating good performance in two-field LN dissection (8). However, for most operators, it is often a difficult decision to remove specific LN stations when performing MIE. Stage T1 esophageal cancer is still early localized lesions, and most stage T1a patients are treated by endoscopic submucosal dissection (ESD), while stage T1b patients are treated predominantly by surgery. Biologically, stage T1b esophageal cancer can be detected early, and the probability of LN metastasis is low. The esophagus has abundant lymphatic channels in the submucosa, and there remains a certain rate of $\mathrm{LN}$ metastasis. Our current study retrospectively analyzed the previous open surgeries performed in our center to investigate the $\mathrm{LN}$ metastasis of 
stage T1b mid-thoracic esophageal cancer and explore the value of $\mathrm{LN}$ dissection for different $\mathrm{LN}$ stations, so as to inform intraoperative $\mathrm{LN}$ dissection (or not).

During 3FD, the difficulty of $\mathrm{LN}$ dissection varies among different sites due to the differences in anatomical locations. For example, LNs that can be routinely dissected (e.g., upper, middle, and lower paraesophageal LNs, paracardiac LNs, subcarinal LNs, LNs near the lesser curvature of stomach, and LNs adjacent to the left gastric artery) are generally easy to remove. In contrast, dissection of LNs such as the right and left RLN LNs and supraclavicular LNs are more challenging and can be associated with a high incidence of complications. Therefore, in our current study, the levels of LN metastasis and the corresponding EI values were observed to determine the necessity and extent of dissection. It was found that stage T1b mid-thoracic ESCC had the highest rate of metastasis in the paracardiac LNs (4.3\%), followed by the right RLN LNs (2.8\%) and the left gastric artery LNs (2.8\%). Therefore, dissection of abdominal LNs is valuable for patients with stage $\mathrm{T} 1 \mathrm{~b}$ ESCC. Also, metastasis to the right RLN LNs may be associated with abundant longitudinal lymphatic drain in the submucosa of the esophagus (9).

We further explored the survival rate of patients who underwent $\mathrm{LN}$ dissection, and found that the 5-year survival rate was only $28.6 \%$ in patients who received paracardiac $\mathrm{LN}$ dissection, but reached $80 \%$ in those who had undergone right RLN LN dissection. Furthermore, the EI value of RLN LN dissection was also significantly higher than that of paracardiac LN dissection. Numerous studies have demonstrated that right RLN LNs (including intrathoracic and right medial cervical LNs) are the sites with the highest LN metastasis rate, and dissection in these sites is more likely to benefit esophageal cancer patients $(10,11)$. Therefore, right RLN LN dissection is mandatory for stage T1b ESCC patients. In addition, the EI value of supraclavicular LN dissection was low for our subjects. Thus, modern two-field LN dissection, including RLN LN dissection, may be sufficient for most midthoracic ESCC patients, and the value of complete 3FD is not convincing.

In conclusion, right RLN LNs have a metastasis rate only lower than that of the paracardiac LNs, but could be the most valuable location for performing dissection. Modern two-field LN dissection, including right RLN LN dissection, is mandatory for stage T1b ESCC. The value of complete $3 \mathrm{FD}$ for stage T1b ESCC requires further investigation.

\section{Acknowledgments}

Funding: Supported by the Fujian Provincial Health and Family Planning Research Talent Training Project (2018-CXB-3). Research topic: Study on the application of inflatable mediastinal lumpectomy combined with laparoscopy for radical treatment of esophageal cancer (principal investigator: LIU Shuoyan).

\section{Footnote}

Reporting Checklist: The authors have completed the STROBE reporting checklist. Available at https://dx.doi. org/10.21037/jgo-21-339

Data Sharing Statement: Available at https://dx.doi. org/10.21037/jgo-21-339

Conflicts of Interest: All authors have completed the ICMJE uniform disclosure form (available at https://dx.doi. org/10.21037/jgo-21-339). The authors have no conflicts of interest to declare.

Ethical Statement: The authors are accountable for all aspects of the work in ensuring that questions related to the accuracy or integrity of any part of the work are appropriately investigated and resolved. The present study was approved by the Fujian Cancer Hospital Ethics Committee (YKT2021-011-01), and the informed consent was signed by all patients before undergoing surgery. The study was conducted in accordance with the Declaration of Helsinki (as revised in 2013).

Open Access Statement: This is an Open Access article distributed in accordance with the Creative Commons Attribution-NonCommercial-NoDerivs 4.0 International License (CC BY-NC-ND 4.0), which permits the noncommercial replication and distribution of the article with the strict proviso that no changes or edits are made and the original work is properly cited (including links to both the formal publication through the relevant DOI and the license). See: https://creativecommons.org/licenses/by-nc-nd/4.0/.

\section{References}

1. Jemal A, Bray F, Center MM, et al. Global cancer statistics. CA Cancer J Clin 2011;61:69-90.

2. Sohda M, Kuwano H. Current Status and Future 
Prospects for Esophageal Cancer Treatment. Ann Thorac Cardiovasc Surg 2017;23:1-11.

3. Sasako M, McCulloch P, Kinoshita T, et al. New method to evaluate the therapeutic value of lymph node dissection for gastric cancer. Br J Surg 1995;82:346-51.

4. Takeuchi H, Miyata $\mathrm{H}$, Gotoh $\mathrm{M}$, et al. A risk model for esophagectomy using data of 5354 patients included in a Japanese nationwide web-based database. Ann Surg 2014;260:259-66.

5. Law S, Wong J. Current management of esophageal cancer. J Gastrointest Surg 2005;9:291-310.

6. Yasuda T, Yano M, Miyata H, et al. Evaluation of dysphagia and diminished airway protection after three-field esophagectomy and a remedy. World J Surg 2013;37:416-23.

7. Nakamura M, Kido Y, Hosoya Y, et al. Postoperative gastrointestinal dysfunction after 2-field versus 3-field lymph node dissection in patients with esophageal cancer. Surg Today 2007;37:379-82.

Cite this article as: Chen $\mathrm{X}$, Chen $\mathrm{Y}$, Wang F, Liu S, Chen P, $\mathrm{He} \mathrm{H}$. Clinical value of lymph node dissection for stage T1b mid-thoracic esophageal squamous cell carcinoma. J Gastrointest Oncol 2021;12(4):1223-1227. doi: 10.21037/jgo21-339
8. Mann C, Berlth F, Hadzijusufovic E, et al. Minimally invasive esophagectomy: clinical evidence and surgical techniques. Langenbecks Arch Surg 2020;405:1061-7.

9. Mizutani M, Murakami G, Nawata S, et al. Anatomy of right recurrent nerve node: why does early metastasis of esophageal cancer occur in it? Surg Radiol Anat 2006;28:333-8.

10. Li H, Yang S, Zhang Y, et al. Thoracic recurrent laryngeal lymph node metastases predict cervical node metastases and benefit from three-field dissection in selected patients with thoracic esophageal squamous cell carcinoma. J Surg Oncol 2012;105:548-52.

11. Udagawa $H$, Ueno $M$, Shinohara $H$, et al. The importance of grouping of lymph node stations and rationale of threefield lymphoadenectomy for thoracic esophageal cancer. J Surg Oncol 2012;106:742-7.

(English Language Editor: A. Kassem) 